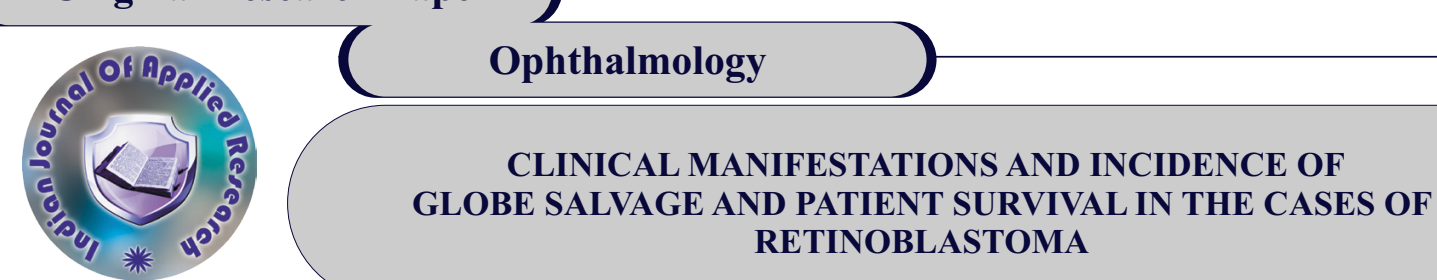

\section{Dr. Bansari} Sorathiya*

Dr. Wilhemina Chauhan
Resident Doctor, M\&J Institute of Ophthalmology, Civil Hospital, Ahmedabad. ${ }^{*}$ Corresponding Author

\title{
ABSTRACT
} PURPOSE: To study clinical characteristics of Retinoblastoma and analyze the occurrences of patient survival and globe salvation.

METHODS: This is a prospective and non-randomized study involving retinoblastoma patients at a tertiary institute from May, 2017 to May, 2019. All patients underwent comprehensive medical assessment and illness management investigations. The given treatment was recorded, including focal therapy, enucleation and chemotherapy.

RESULTS: Leukocoria was the foremost common presentation (60.68\%) followed by proptosis (12.3\%). The patient survival rate was $87.7 \%$ whereas globe salvation was at $23.2 \%$ (19 eyes).

CONCLUSION: Most patients came for clinical examination for first time at a later stage of disease, having high risk clinical features such as positive family history, hyphema, and large tumor volume. More awareness and early stage diagnosis are of prime importance in order to achieve globe salvation in retinoblastoma.

\section{KEYWORDS : Enucleation, Leukocoria, Proptosis}

\section{INTRODUCTION:}

Study of Retinoblastoma demands urgent attention because it is the most common primary intraocular malignancy of childhood [1]. 9-10 $\%$ of all pediatric cancer patients $(<14$ years $)$ in India have retinoblastoma [2].

At presentation, Leukocoria is the most common symptom. Other symptoms are redness, poor vision, proptosis, or squint. Retinoblastoma is a significant challenge, particularly in the developing nations, despite all the recent improvements in the diagnosis. More than half of its patients die from this condition, and those seeking treatments are usually at an advanced stage [3].

Complex and unclear protocols of treatment have resulted into even worse survival rates in the cases of extraocular retinoblastoma. Various studies from India have revealed that a large population of children were at advanced stage at presentation, having mortality as high as $24 \%$ [4]. Even though it may be curable, it's the early diagnosis and appropriate therapy that decide the survival prognosis. Therapy aims consist of saving the patients' life, salvaging globe and preserving vision whenever possible.

\section{OBJECTIVE:}

The goal of this research is to determine the clinical characteristics and outcome of the prescribed therapy in the cases of Retinoblastoma with regard to patient survival and globe salvation.

\section{MATERIALS AND METHODOLOGY: \\ Study Type: A Prospective Study}

Study Site: M \& J Western Regional Institute of Ophthalmology, Civil Hospital Ahmedabad.

Sample Size: 65 patients (82 Eyes)

Inclusion criteria: Newly diagnosed cases of Retinoblastoma.

Exclusion criteria: Cases lost to follow up

Duration: May'17-May'19

Based on clinical presentation, histopathological characteristics, and radiological evaluation, we classified the tumors into intraocular and extraocular categories. For that, we used "International Intraocular Retinoblastoma Classification" (IIRC) [6] and "International Retinoblastoma Staging System" [7].

After taking informed consent from a guardian, a careful ocular and systemic history was taken for each patient including name, age, sex, address, presenting complaints and their duration, birth history and family history of similar complaints. Clinical features including leukocoria, proptosis, conjunctival hyperemia or chemosis, squint, lid swelling and hyphema were noted. Under general anesthesia, indirect ophthalmoscopy was performed to examine the fundus. MRI was performed to study tumor characteristics and the extent of invasion.

\section{CLASSIFICATION:}

We followed the classification system given by Shields, J.A., \& Shields, C.L. in [11], which is as follows:

Group $\mathrm{A}: \mathrm{Rb}<=3 \mathrm{~mm}$, at least $3 \mathrm{~mm}$ from the foveolar and $1.5 \mathrm{~mm}$ from optic nerve. No seeding. [11]

Group B: Eyes with no vitreous or sub retinal seeding and retinal tumor of any size or location not included in Group A. Small cuff of sub retinal fluid $<=5 \mathrm{~mm}$ from tumor margin. [11]

Group C: Eyes with focal vitreous or sub retinal seeding and discrete tumor of any size or location. Seeding must be local, fine and limited so as to be theoretically treatable with a radioactive plaque. Up to one quadrant subretinal fluid may be present. [11]

Group D: Eyes with diffused vitreous or subretinal seeding and/or massive, non-discrete endophytic or exophytic disease. Seeding more extensive than GroupC. Retinal detachment more than one quadrant. [11]

Group E: Massive $\mathrm{Rb}$ with anatomic or functional destruction with one or more of the following:

- Neovascular Glaucoma

- Massive Intraocular hemorrhage

- Aseptic orbital cellulitis

- Tumor anterior to anterior vitreous face

- Tumor touching lens

- Diffuse infiltrating tumor

- Phthisis or Pre-phthisis [11]

$(\mathrm{Rb}=$ Retinoblastoma $)$

Extraocular: Tumor with orbital extension and/or optic nerve invasion examined clinically and radiologically on MRI.

Metastasis: Intracranial extension and spread to other distant organs.

TREATMENT PROTOCOL:

Treatment protocols given in [12] were followed in our cases of 
Retinoblastoma.

\begin{tabular}{|l|l|}
\hline Tumor size \& location & Treatment Protocol \\
\hline $\begin{array}{l}<3 \mathrm{~mm} \text { in size, visually } \\
\text { non crucial area }\end{array}$ & $\begin{array}{l}\text { Focal therapy } \\
\text { (laser photocoagulation) }\end{array}$ \\
\hline $\begin{array}{l}\text { Larger tumor, visually } \\
\text { crucial area }\end{array}$ & $\begin{array}{l}\text { 6 cycle chemoreduction and } \\
\text { sequential aggressive focal therapy }\end{array}$ \\
\hline Small residual tumor & Focal therapy(Laser photocoagulation) \\
\hline Large residual tumor & $\begin{array}{l}\text { Bilateral-Plaque brachytherapy /EBRT } \\
\text { Unilateral-Enucleation }\end{array}$ \\
\hline
\end{tabular}

A. Intraocular tumor. IIRC Group A-C, Unilateral or Bilateral. (TABLE1)

B. Intraocular tumor, IIRC Group D, Unilateral/Bilateral:

1. High dose chemotherapy and sequential aggressive focal therapy

2. Primary enucleation if unilateral, especially in eyes with no visual prognosis

C. Intraocular tumor, IIRC Group E, Unilateral/Bilateral:

1. Primary enucleation

2. Dose adjusted adjuvant chemotherapy if high risk factors on histopathological evaluation like scleral infiltration, extraocular extension, and optic nerve extension.

3. Baseline systemic evaluation for metastasis

D. Extraocular tumor, International Staging, Stage 3A

1. Baseline systemic evaluation for metastasis

2. High dose chemotherapy for 3-6 cycles, followed by enucleation/extended enucleation and continued high dose chemotherapy for 12 cycles

E. Regional Lymph Node Metastasis, International Staging, Stage 3B: Baseline evaluation for systemic metastasis, consider for high dose chemotherapy for 12 cycles

F. Systemic Metastasis, International Staging, Stage 4: Palliative therapy in discussion with family, High dose chemotherapy for 12 cycles.

The advised chemotherapy regimen was VEC, which comprises of vincristine $(1.5 \mathrm{mg} / \mathrm{m} 2)$, etoposide $(150 \mathrm{mg} / \mathrm{m} 2)$, and carboplatin(360 $\mathrm{mg} / \mathrm{m} 2$ ), all administered on the same day.

After the therapy, follow-up examination was done every three months for the first two years, followed by four to six monthly examinations based on the initial stage of disease. The outcome was defined in terms of survival of patients and globe salvation rate.

\section{RESULTS:}

A total of 65 patients ( 82 eyes) were studied, among them $29(45 \%)$ were female and $36(55 \%)$ were male. Retinoblastoma was bilateral in 17 patients $(26 \%)$ and unilateral in 48 patients (74\%). 50 patients belonged to rural areas, while 15 came from urban areas. Family history of similar complaint was positive in 6 patients $(3.9 \%)$. The Median age at presentation was 24 months, and the Mean age was 28.8 months. (31.7 for Unilateral and 20.4 for Bilateral). The Range was 1.5-72 months

Table 2: Clinical Features On Presentation.

\begin{tabular}{|l|l|}
\hline Symptoms & \%(percentage) \\
\hline Leukocoria & 60.68 \\
\hline Proptosis & 12.3 \\
\hline Squint & 8.2 \\
\hline Hyphema & 6.56 \\
\hline Secondary Glaucoma & 4.92 \\
\hline Orbital Cellulitis & 4.1 \\
\hline Fungating mass & 3.28 \\
\hline Others & 1.64 \\
\hline
\end{tabular}

\section{DISCUSSION:}

The present study reports 65 patients who were studied for sociodemographic, symptomatology, treatment and outcome from a tertiary health care center. At presentation, the mean age of our patients was 28.8 months, which is comparable to the range of 29-33 months in previous reports [10] from India. Out of 65 patients, 57(87.69\%) were presented with advanced stage (GroupD/E of IIRC, extraocular disease), in which globe salvation was very difficult. Median time lag between onset of symptom and diagnosis was 3 months. The most common sign was leukocoria with a presence rate of $60.68 \%$ followed by proptosis with $12.3 \%$. These stats are also in accordance with a previous study [10] wherein they were $83 \%$ and $17 \%$ respectively. Usha Singh et al. [10] reported that family history of Retinoblastoma was present in $4.09 \%$, which is comparable to $3.9 \%$ in our study. Chawla B et al. [4] reported $78 \%$ of advanced group D/E disease and $15.7 \%$ of extraocular disease while only 7 patients $(10.76 \%)$ were presented to us with early disease (IIRC Group A-C), the rest 58 patients $(89.23 \%)$ were with advanced disease and $15.85 \%$ had extraocular disease. The globe salvation rate was $23.2 \%$ (19 eyes) while patient survival was $87.7 \%$.

Out of 65 patients (82 eyes) 26 patients underwent enucleation as primary treatment, 32 received chemotherapy and 7 patients received Laser photocoagulation. Out of the 26 patients who had received Enucleation as primary treatment, 18 received Adjuvant Chemotherapy as secondary treatment (because their histopathological findings showed high risk factors) and 7 received adjuvant chemotherapy and radiotherapy, both as secondary mode of treatment. Of the 32 patients who had received chemoreduction as primary treatment, 19 received enucleation and 8 patients received laser photocoagulation as their secondary treatments.

Table 3: Analysis Of Unilateral Vs Bilateral Cases

\begin{tabular}{|c|c|c|c|c|c|c|c|c|}
\hline \multirow[t]{2}{*}{ Disease type } & \multirow[t]{2}{*}{ No. of Eyes } & \multirow{2}{*}{$\begin{array}{c}\text { Globe } \\
\text { Salvage Rate }(\%)\end{array}$} & \multicolumn{3}{|c|}{ Unilateral } & \multicolumn{3}{|c|}{ Bilateral } \\
\hline & & & Eyes & Globe salvation & Deaths & Eyes & Globe salvation & Deaths \\
\hline Group A & 1 & 100 & 0 & - & - & 1 & 1 & 0 \\
\hline Group B & 12 & 100 & 1 & 1 & 0 & 11 & 10 & 1 \\
\hline Group C & 11 & 82 & 3 & 3 & 0 & 8 & 6 & 2 \\
\hline Group D & 22 & 0 & 17 & 0 & 0 & 5 & 0 & 0 \\
\hline Group E & 22 & 0 & 16 & 0 & 2 & 6 & 0 & 3 \\
\hline Extraocular & 13 & 0 & 10 & 0 & 2 & 3 & 0 & 1 \\
\hline Total & 82 & & & & & & & \\
\hline
\end{tabular}

\section{CONCLUSION:}

Delayed presentation in Retinoblastoma cases is a serious matter of concern. Our results show that the most common presenting sign was leukocoria followed by proptosis. Lack of awareness about the disease, lower socioeconomic class and inadequate facilities at primary and secondary health care centers play a major role in mortality and morbidity of these patients. Early counselling and treatment of the disease and convincing the patients to accept the treatments including enucleation can bring significant improvement in patient survival.

\section{STATEMENT OF ETHICS:}

Approval of the Institutional ethics committee was obtained for this study. Informed consent was obtained from parents for their ward's inclusion in the study during their treatment.
REFERENCES:

[1] Lennox, E. L., Draper, G. J., \& Sanders, B. M. (1975). Retinoblastoma: a study of natural history and prognosis of 268 cases. Bmj, 3(5986), 731-734. doi: 10.1136/bmj.3.5986.731

[2] Arora, R. S., Eden, T., \& Kapoor, G. (2009). Epidemiology of childhood cancer in India. Indian Journal of Cancer, 46(4), 264. doi: 10.4103/0019-509x.55546

[3] Shields, C. L., \& Shields, J. A. (2004). Diagnosis and Management of Retinoblastoma. Cancer Control, 11(5), 317-327. doi: 10.1177/107327480401100506

[4] Chawla, B., Hasan, F., Azad, R., Seth, R., Upadhyay, A. D., Pathy, S., \& Pandey, R. (2015). Clinical presentation and survival of retinoblastoma in Indian children. British Journal of Ophthalmology, 100(2), 172-178. doi: 10.1136/bjophthalmol-2015-306672

[5] Albert, D. M., Miller, J. W., \& Jakobiec, F. A. (2008). Albert \& Jakobiecs Principles and practice of ophthalmology. Philadelphia, PA: Saunders Elsevier.

[6] Linn Murphree, A. (2005). Intraocular Retinoblastoma: the Case for a New Group Classification. Ophthalmology Clinics of North America, 18(1), 41-53. doi: 10.1016/j.ohc.2004.11.003

[7] Chantada, G., Doz, F., Antoneli, C. B., Grundy, R., Stannard, F. C., Dunkel, I. J., Zucker, J.-M. (2006). A proposal for an international retinoblastoma staging system. Pediatric Blood \& Cancer, 47(6), 801-805. doi: 10.1002/pbc.20606

[8] Chantada, G., Luna-Fineman, S., Sitorus, R. S., Kruger, M., Israels, T., Leal-Leal, C., 
Doz, F. (2013). SIOP-PODC recommendations for graduated-intensity treatment of retinoblastoma in developing countries. Pediatric Blood \& Cancer, 60(5), 719-727. doi: $10.1002 /$ pbc. 24468

[9] Sitorus, R. S., Moll, A. C., Suhardjono, S., Simangunsong, L. S., Riono, P., Imhof, S., \& Völker-Dieben, H. J. M. (2009). The Effect of Therapy Refusal Against Medical Advice in Retinoblastoma Patients in a Setting Where Treatment Delays are Common. Ophthalmic Genetics, 30(1), 31-36. doi: 10.1080/13816810802464320

[10] Singh, U., Katoch, D., Kaur, S., Dogra, M. R., Bansal, D., \& Kapoor, R. (2017). Retinoblastoma: A Sixteen-Year Review of the Presentation, Treatment, and Outcome from a Tertiary Care Institute in Northern India. Ocular Oncology and Pathology, 4(1), 23-32. doi: 10.1159/000477408

[11] Shields, J. A., \& Shields, C. L. (2016). Intraocular tumors: an atlas and textbook. Philadelphia, PA: Wolters Kluwer.

[12] Honavar, S. G., PM, F. J., Ali, M. K., Vemuganti, G. P., \& Reddy, V. A. Retinoblastoma: They Live and See! (2012, July). PDF. All India Ophthalmological Society. New Delhi. 